\title{
La viscosupplémentation pour le traitement de la gonarthrose prise en charge par l'assurance obligatoire des soins
}

La viscosupplémentation a été admise le $1^{\text {er juil- }}$ let 2002, pour une durée limitée, aux conditions suivantes: elle doit être soumise à une étude d'évaluation et employée pour soigner des patients entravés dans leur liberté de mouvement par une gonarthrose douloureuse (termes médicaux: gonarthrose symptomatique, gêne fonctionnelle), sur lesquels les analgésiques ou d'autres traitements conservateurs n'agissent plus ou sont contre-indiqués. L'objectif à long terme du traitement est de retarder autant que possible la nécessité de recourir à une prothèse (ordonnance sur les prestations de l'assurance des soins [OPAS], annexe 1, chap. 1.3).

Après avoir pris connaissance de la recommandation de la Commission fédérale des prestations (CFP), le Département fédéral de l'intérieur (DFI) a explicitement lié cette décision d'admission à la condition que l'évaluation se fasse dans le cadre d'une étude contrôlée randomisée. Ladite étude, baptisée SVISCOT (pour Swiss Viscosupplementation Trial), sera lancée dans toute la Suisse entre décembre 2002 et mars 2003, selon les décisions prises par les commissions cantonales d'éthique compétentes.

A dater du $1^{\text {er }}$ janvier 2003, le texte relatif à la viscosupplémentation dans l'annexe 1 de l'OPAS est complété comme suit:

- Traitements entrepris dans le cadre de l'étude suisse randomisée et contrôlée SVISCOT pour l'évaluation comparative clinique et économique de la viscosupplémentation.

- Un remboursement forfaitaire est convenu pour le traitement de viscosupplémentation administré dans le cadre de l'étude SVISCOT.
Dès cette date, le traitement n'est donc plus remboursé par l'assurance-maladie sociale (assurance obligatoire des soins) que s'il est intégré dans SVISCOT. En dehors de ce cadre, aucun remboursement ne sera possible à partir du $1^{\text {er }}$ janvier 2003.

Entre le $1^{\text {er }}$ juillet et le 31 décembre 2002, soit durant la phase de mise en place de SVISCOT, les frais de traitement par viscosupplémentation de la gonarthrose symptomatique avec gêne fonctionnelle sont remboursés par les caissesmaladie dans le cadre de l'assurance obligatoire des soins, sans demande de prise en charge, sur la base des conventions tarifaires en vigueur (réglementation transitoire).

\section{Nota bene}

Actuellement, seuls trois produits, Orthovisc ${ }^{\circledR}$, Ostenil ${ }^{\circledR}$ und Synvisc ${ }^{\circledR}$, satisfont à la condition de l'évaluation. La viscosupplémentation à l'aide d'autres produits ne doit donc pas être prise en charge par les assureurs. La viscosupplémentation pour le traitement de la gonarthrose a été admise en Suisse au nombre des prestations médicales; étant donné l'absence d'effet pharmacologique, les produits ne reçoivent pas d'autorisation de mise sur le marché de Swissmedic et ne sont donc pas non plus inscrits sur la liste des spécialités (LS). Le montant du remboursement des prestations (prise en charge forfaitaire) doit donc être fixé dans le cadre des négociations tarifaires avec santésuisse.

Voir aussi le site web de l'étude: www.sviscot.ch. 